\title{
Novel indel Mutation in the GDF5 Gene Is Associated with Brachydactyly Type C in a Four-Generation Turkish Family
}

\author{
Z.O. Uyguner ${ }^{a} \quad$ M. Kocaoğlub ${ }^{b}$ G. Toksoy ${ }^{a} \quad$ S. Basaran ${ }^{a} \quad$ H. Kayserili ${ }^{a}$ \\ Departments of ${ }^{\mathrm{a}}$ Medical Genetics and ${ }^{\mathrm{b}}$ Orthopedics, Istanbul Medical Faculty, Istanbul University, Istanbul, Turkey
}

\section{Key Words}

Brachydactyly $\cdot G D F 5 \cdot$ indel mutation

\begin{abstract}
Heterozygous loss-of-function mutations of GDF5 are reported to cause hypoplasia/aplasia of certain skeletal elements (brachydactyly), and heterozygous gain-of-function mutations, occurring either on the gene itself or through the loss of its inhibitor noggin, result in joint fusion (symphalangism). We present here the clinical and molecular investigation of a family with disproportionate shortness of the second and third fingers which comprises 9 variably affected members spanning 4 generations. In this study, we performed clinical and radiographical examinations of 2 patients of this family, sequencing of GDF5 and 3D protein modeling of the wildtype and mutated polypeptide to predict the structural alteration. Diagnoses were compatible with familial brachydactyly type C. GDF5 analysis revealed a novel heterozygous in-frame indel mutation (c.803 827del25ins25), involving the propeptide domain of GDF5 that alters the number of random coil and beta-strand structures, creating a 1-turn-helix at the mutated site. The mutation described here is the second indel reported in GDF5. The previously published homozygous indel mutation affected the TGF-beta like domain and was associated with Du Pan
\end{abstract}

\section{KARGER}

E-Mail karger@karger.com

www.karger.com/msy syndrome. The novel mutation reported here presents further allelic heterogeneity and a probable intrafamilial variable clinical expressivity of GDF5.

(c) 2014 S. Karger AG, Base

Hereditary nonsyndromic brachydactyly type C (BDC; MIM 113100) is recognized by the brachymesophalangy of the second, third and fifth fingers with hypersegmentation usually of the second and/or the third finger and shortening of the first metacarpal (fig. 1). As a rule, the fourth finger is not affected and is generally perceived as the longest digit in BDC. Accompanying skeletal and nonskeletal features, such as short stature, hip dysplasia, Madelung deformity, and talipes valgus, are also occasionally reported [Tentamy and Aglan, 2008; Mundlos, 2009]. The causative gene, growth differentiation factor 5 (GDF5) also named cartilage-derived morphogenetic protein-1 (CDMP1), located on 20q11.2, was identified by Polinkovsky et al. [1997]. It is a member of the TGF-beta superfamily, which comprises regulators of cell growth and differentiation in both embryonic and adult tissues by activating cell-specific receptors for transducing signals to the target genes [Tsumaki et al., 1999]. Human GDF5 encompasses 501 amino acids in length (NP_000548.1), with amino acids 1-27 


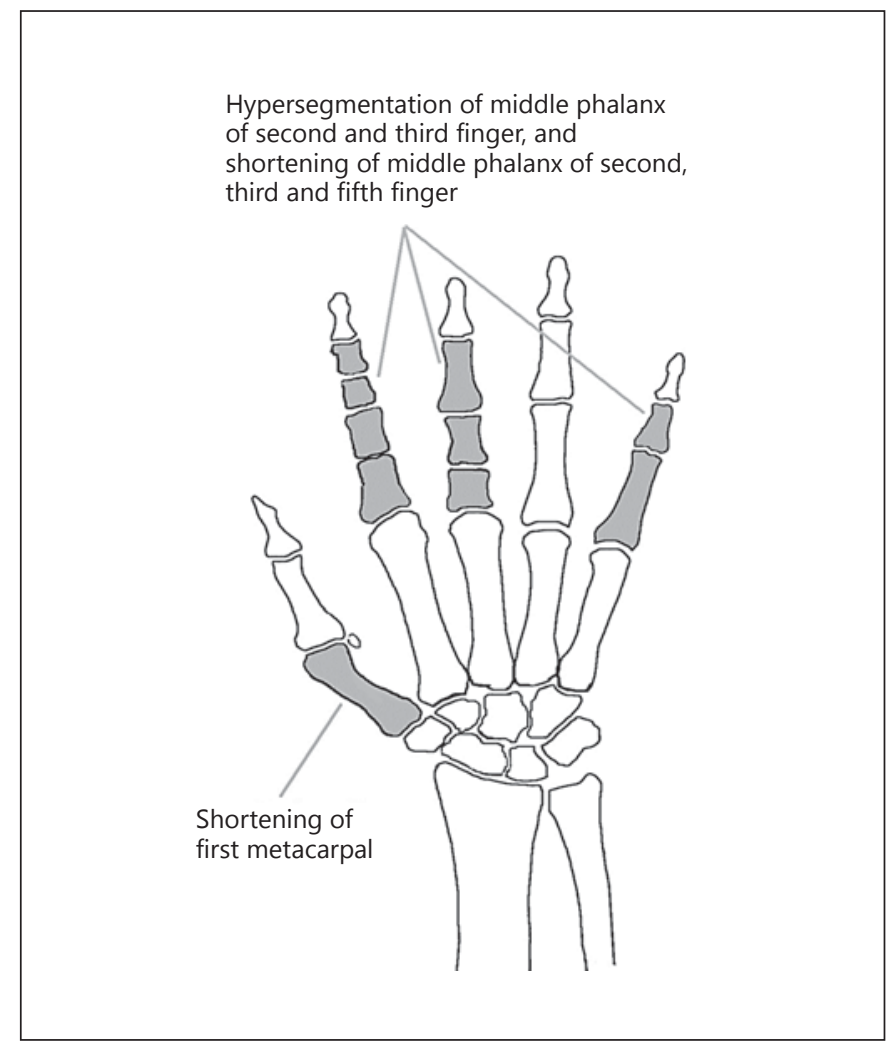

Fig. 1. Schematization of human hand skeleton showing the possibly affected areas (shaded) in brachydactyly type C. coding for the signal peptide, 28-381 coding for the propeptide and 382-501 coding for the TGF-beta like domain (UniProt KB_P43026). The protein harbors 3 disulfide bonds to form intersubunit ties to dimerize for activation in addition to hydrophobic interactions [Massaqué, 1998]. A number of functional investigations suggested that loss-of-function mutations are responsible for a decrease in GDF5 activity, evident in the underdevelopment and hypersegmentation of the digits in hand and foot resulting in brachydactyly. Conversely, an increase in GDF5 activity, probably due to an alteration of its active site to a resistant form against inhibition by noggin, influences fusing of the joints resulting in symphalangism of the digits in hand and foot [Seemann et al., 2005, 2009; Yang et al., 2008].

Presently, 37 different mutations have been described in the GDF5 gene: 17 are associated with BDC, while 20 cause 7 other allelic phenotypes: brachydactyly type A1, C (MIM 615072); brachydactyly type A2 (MIM 112600); chondrodysplasia, Grebe type (MIM 200700); Du Pan syndrome (MIM 228900); acromesomelic dysplasia, Hunter-Thompson type (MIM 201250); multiple synostoses syndrome 2 (MIM 610017); and proximal symphalangism, 1B (MIM 615298). Furthermore, 2 regulatory alterations have been associated with susceptibility to osteoarthritis (MIM 612400) (fig. 2A-D). Extensive

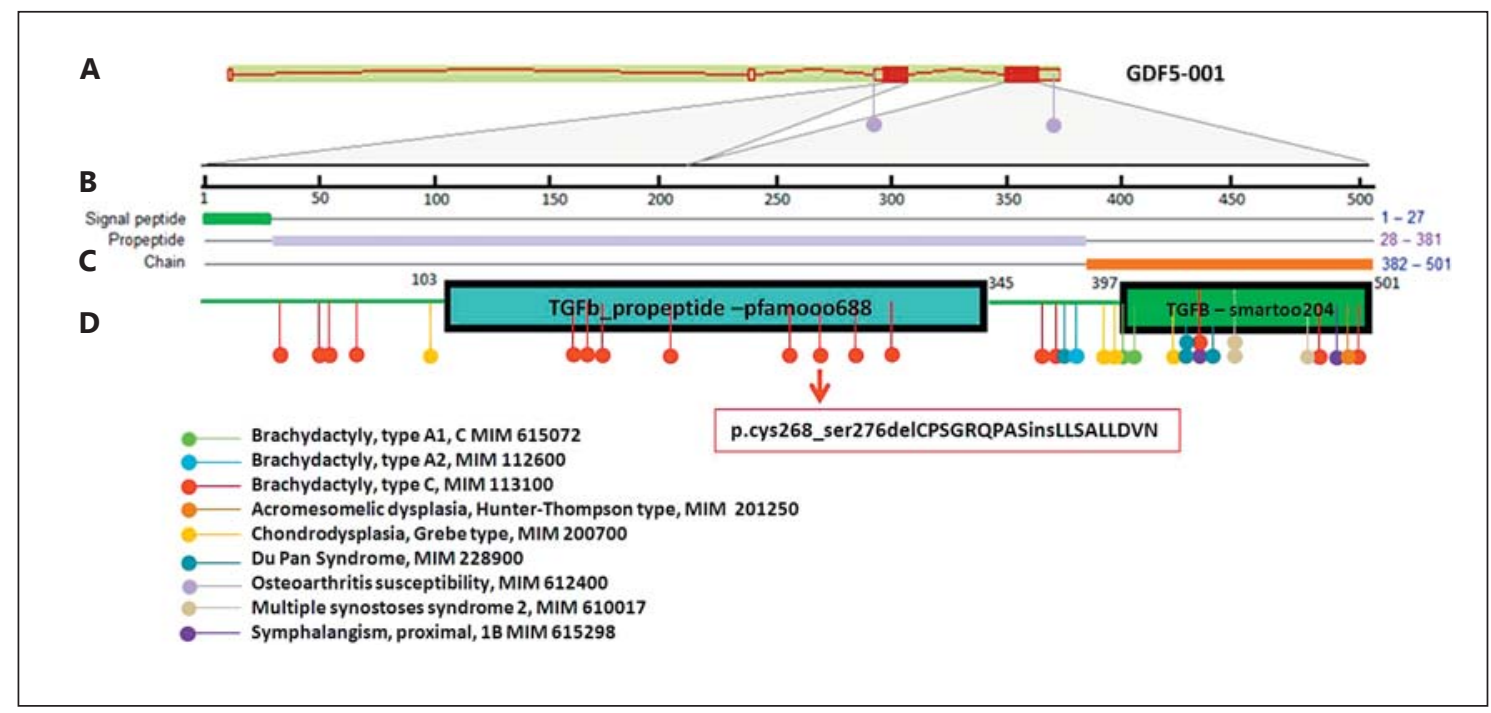

Fig. 2. A Genomic layout of the human GDF5 gene, as displayed in the Ensembl database (http://www.ensembl.org). B Length of the coding regions scaled from base pairs to amino acids, presenting 210 amino acids encoded by exon 1 and 291 amino acids encoded by exon 2. C Regions of the human GDF5 protein, according to molecular processing (http://www.uniprot.org/uniprot/P43026). D Domains of the human GDF5 protein (http://pfam.sanger.ac. uk/protein/P43026). Locations of mutations associated with 9 OMIM entries are pointed on the domains. The mutation identified in this study is presented in the box indicated by an arrow. 
genotype-phenotype correlations have been discussed by Gutiérrez-Amavizca et al. [2012].

We present here clinical and molecular findings of a family with $\mathrm{BDC}$, due to a novel indel mutation in the GDF5 gene.

\section{Case Reports}

A female 28-year-old patient of Turkish Caucasian origin was referred by an orthopedic surgeon for genetic consultation due to the abnormal appearance of fingers on both hands (fig. 3A, IV:1). Her maternal 52-year-old uncle was also consulted for affected sta- tus (fig. 3A, III:1). A review of the family's history revealed the presence of 6 severely (black filled symbols in fig. 3A) and 3 mildly (black checkered symbols in fig. 3A) affected individuals.

The medical history and physical examination of both patients were found to be within normal limits: they neither exhibited a short stature nor other skeletal manifestations such as joint problems other than the distinct phenotype of their hands (fig. 3B). Radiographs of patient IV:1 revealed disproportionate shortening of the second and third fingers and hypersegmentation of the second and third fingers' proximal phalanges, giving a pseudo 4-phalangeal appearance (fig. 3C, D). Hand radiographs of patient III:1 presented mildly short middle and distal phalanges of second, third and fifth fingers (fig. 3E).

The clinical and radiological findings of patients III:1 and IV:1 were compatible with familial brachydactyly type C.
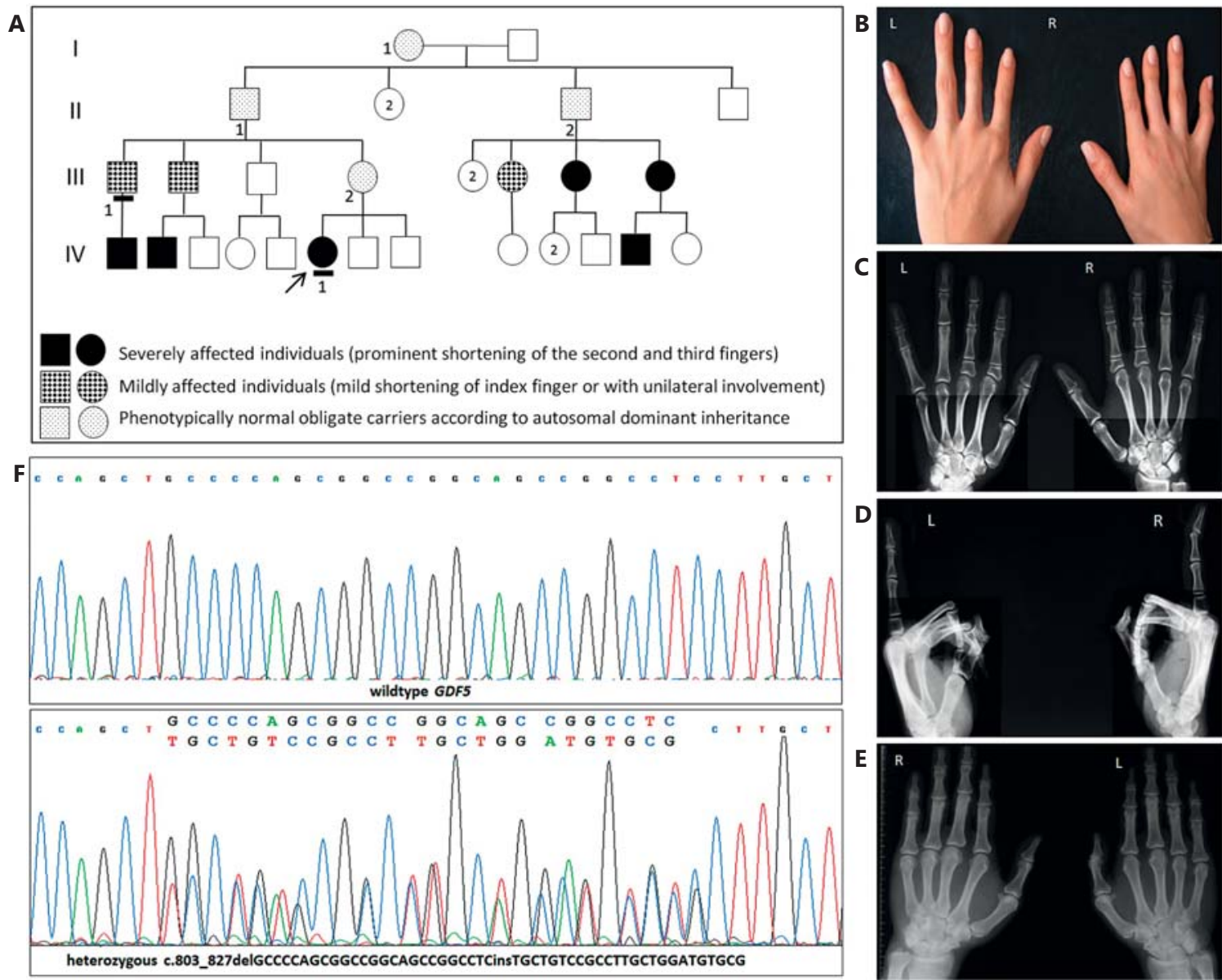

Fig. 3. A Pedigree of the BDC family. The 2 clinically, radiographically and molecularly investigated individuals are underlined. B Both hands of patient IV:1, presenting abnormal disproportionate shortening of the second and third fingers bilaterally. C Anteroposterior hand radiographs of patient IV:1, exhibiting hypersegmentation of the second and third fingers' proximal phalanges. D Lateral hand radiographs of patient IV:1. The second fingers show hypersegmentation of proximal phalanges with pseudo 4-phalangeal appearance. Middle and distal phalanges of the second fingers are mildly shortened. E Radiographs of patient III:1, presenting mildly shortened second and third fingers due to hypoplastic middle and distal phalanges. F Sequencing electropherogram of the wildtype and mutated GDF5 gene. 


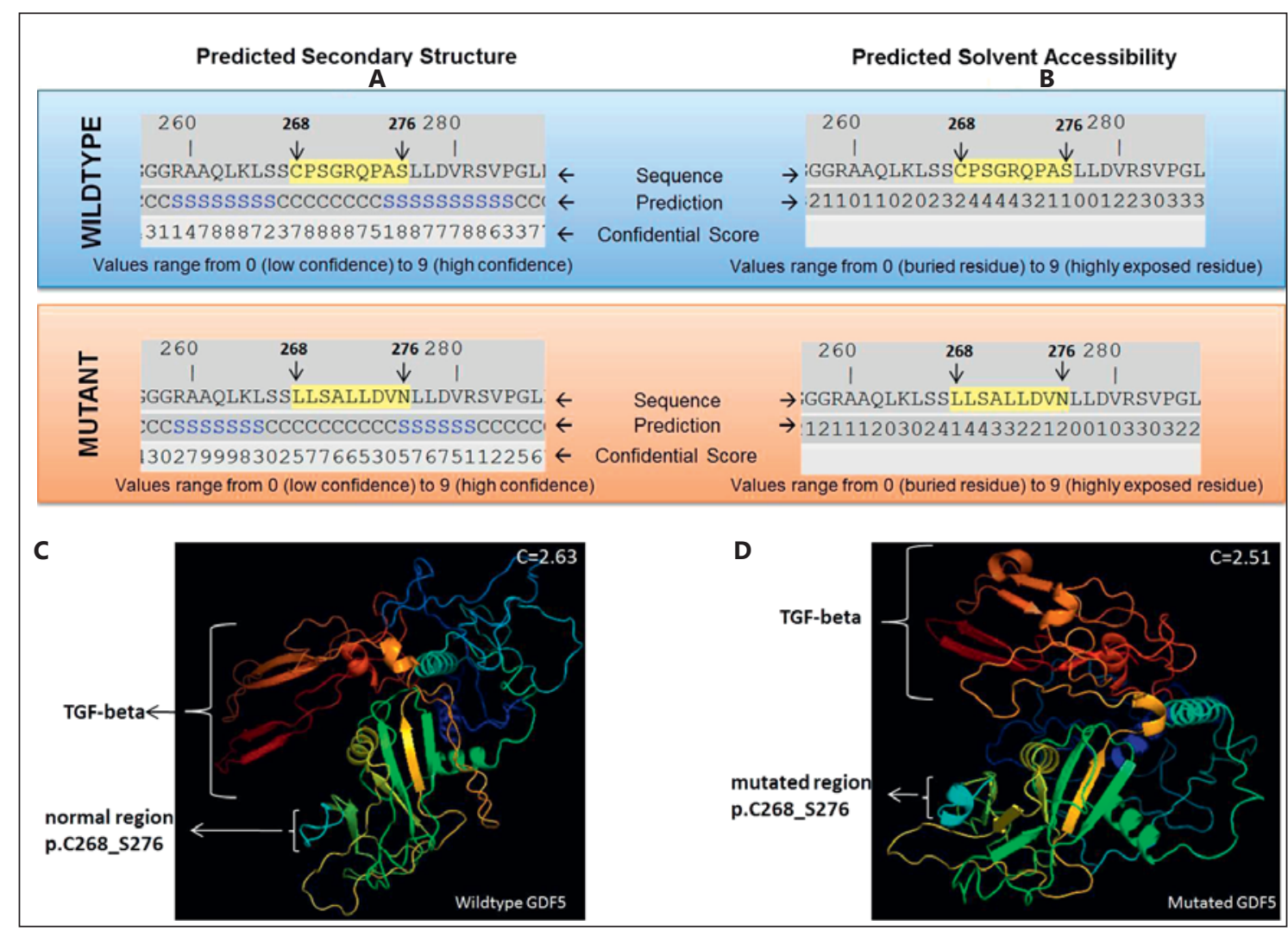

Fig. 4. Results of predicted secondary structure (A) and predicted solvent accessibility (B) of wildtype and mutated GDF5. Numbers on the very top show the corresponding residue numbers. The amino acids highlighted in yellow are indicating the replaced amino acids. Predicted secondary structures that are shown as 'S' for beta-strands and 'C' for coiled strands with their listed confidential scores (0-9) and predicted values for solvent accessibility (0-9). 3D models of wildtype (C) and mutated GDF5 (D), showing the TGF-beta domain and mutated site of the protein chain. The $\mathrm{C}$ values, which are confidence scores of each predicted model by I-TASSER, are presented on the right top of the models.
Genomic DNA of patients III:1 and IV:1 was isolated from peripheral blood samples. Sequencing of the GDF5 gene (NM_000557.2) revealed a novel heterozygous in-frame indel mutation (c.803_827del25ins25), altering 25 nucleotides located in exon 2 (fig. 3F).

Individuals I:1, II:1, II:2, and III:2, not volunteered to join to this investigation, were normal by history, though were obligate carriers of the indel alteration according to autosomal dominant inheritance (black speckled symbols in fig. 3A). Patient I:1 was considered as an obligate carrier due to the history that her siblings, children and grandchildren had similar hand phenotype. Pedigree analysis was interpreted as autosomal dominant inheritance with a probably decreased penetrance.

An online in silico tool, I-TASSER (http://zhanglab.ccmb. med.umich.edu/I-TASSER/), was used to analyze the predicted structural alterations of the wildtype and mutated GDF5. This program constructs protein conformations based on multiple threading alignments that retrieve templates with similarly folding amino acid sequences from the Local Meta-Threading-Server (http://zhanglab.ccmb.med.umich.edu/LOMETS/) [Zhang, 2008; Roy et al., 2010]. Hydrophobic interactions are the major driving forces in the protein folding and are important in the formation of secondary structure elements such as alpha-helices and betasheets [Chen and Zhou, 2005]. The residues with polar and charged side chains would be hydrophilic with more solvent accessibility than residues with nonpolar side chains. Therefore, alterations of the residues from buried (hydrophobic) or exposed (hydrophilic) states are expected to change the functional stability of the protein. A typical secondary structure prediction by ITASSER contains 3 states: alpha-helix, beta-strand and coiled strand with confidence scores of prediction $(0=$ low, $9=$ high $)$ for each residue [Jones, 1999]. Values of predicted solvent accessibility range from 0 (buried residue) to 9 (exposed residue). The comparison of the predicted secondary structures of the wildtype and mutated GDF5 revealed a lesser number of beta-strands and a higher number of random coiled structures of the mutated protein (fig. 4A) and alterations of the solvent accessibility values on the mutation site (fig. 4B). A 3D prediction model of I-TASSER provided 5 predictions with an individual confidence score which estimates the quality of the predicted models based on the capacity of the threading template alignments and the convergence parameters of the structure assembly simulations. Typically, the 
confidence score of $3 \mathrm{D}$ model ranges between -5 and +2 , where the higher value signifies higher confidence. Among the 5 best 3D models created by the server, the highest confidence score was -2.63 for wildtype and -2.51 for mutant protein to visualize the disparities (fig. 4C, D).

\section{Discussion}

Genes involved in the regulation of chronological and 3-dimensional architectural differentiations and in the development of chondrocytes are essential for the determination of skeletal components. Mutations in the developmentally important genes involved in various bone morphogenic protein pathways are already defined in many isolated and syndromic brachydactylies. Recent classification of the brachydactylies under the term of 'molecular disease families' provides insightful description for these variable and overlapping phenotypes, embracing all the developmental molecular components underlying the disease pathology [Mundlos, 2009].

Isolated shortness of fingers and toes may occur in many different patterns, which are classified under 4 types of brachydactyly groups (A-D), including several subgroups according to their pattern of incomplete skeletal development. Type A encompasses the middle phalanges. Type B addresses the distal phalanges. Type $\mathrm{C}$ describes multiple phalanges with relatively normal digit 4 . Type $\mathrm{D}$ is defined by short distal phalanges of the thumb. Subgroup type $\mathrm{E}$ is manifested by a shortening of the metacarpals.

Upon exclusion of possible syndromic brachydactyly forms, detailed examination of the radiograph confirmed the brachydactyly subtype as type $\mathrm{C}$ in our cases. The novel identified indel mutation affects the potential propeptide domain, replacing the 9 amino acids CPSGRQPAS with LLSALLDVN, including codons 268-276 (p.cys268_ ser276delCPSGRQPASinsLLSALLDVN) staying inframe. Presently, 13 out of 17 mutations associated with BDC in GDF5 are located in the propeptide domain. The general function of the propeptide domains is attributed to the cellular trafficking and cotranslational protein folding, which is important in posttranslational control of gene expression and function [Tang et al., 2003; Hebert and Molinari, 2007]. When the chemical classes of the replaced amino acids are evaluated, general alterations from largely polar groups to nonpolar are revealed, which are further supported by the predicted solvent accessibility values altering from ' 244443211 ' to ' 144332212 '. These alterations are manifested by an increase of the coiled structure from 8 to 10 , causing a 1-turn helix at the mutated site as predicted in the 3D models (fig. 4C, D).

Considering all acquired data, we propose that our 25 -nucleotide indel alteration is a disease-causing mutation in our BDC family.

It has been reported that indel alterations are the second most abundant variations (21\%) observed in the human genome after single nucleotide polymorphisms (79\%). Most of the indel alterations are $2-16$ bp (96\%) in length and the largest reported encompasses $55 \mathrm{bp}$ [Mullaney et al., 2010]. indels where the bp-difference between the deletion and the insertion is a multiple of 3 will preserve the reading frame.

This is the second indel mutation identified in GDF5. The first, c.1309_1319del11ins8, was identified in a homozygous form, replacing 11 nucleotides with 8 nucleotides, staying in frame, substituting amino acids LRSH with RTL in the chain motif encoding the TGF-beta like domain, causing Du Pan syndrome, a rare form of acromesomelic dysplasias [Szczaluba et al., 2005]. indel mutations are not widely reported in single gene disorders. The novel indel mutation described here further emphasizes the allelic heterogeneity of GDF5. Our data also supports the intrafamilial clinical and radiological variability and a probable reduced penetrance in brachydactyly type C families.

\section{Acknowledgement}

The authors would like to thank the patients for their cooperation and assistance.
References
Chen H, Zhou H-X: Prediction of solvent acces- sibility and sites of deleterious mutations from protein sequence. Nucleic Acids Res 33: 3193-3199 (2005).
- Gutiérrez-Amavizca BE, Brambila-Tapia AJ Juárez-Vázquez CI, Holder-Espinasse M, Ma- nouvrier-Hanu S, et al: A novel mutation in $C D M P 1$ causes brachydactyly type $\mathrm{C}$ with 'an- gel-shaped phalanx'. A genotype-phenotype correlation in the mutational spectrum. Eur J Med Genet 55:611-614 (2012).
- Hebert DN, Molinari M: In and out of the ER: protein folding, quality control, degradation, and related human diseases. Physiol Rev 87: 1377-1408 (2007).
-Jones DT: Protein secondary structure prediction based on position-specific scoring matrices. J Mol Biol 292:195-202 (1999).
Massaqué J: TGF-beta signal transduction. Annu Rev Biochem 67:753-791 (1998). 
Mullaney JM, Mills RE, Pittard WS, Devine SE: Small insertions and deletions (INDELs) in human genomes. Hum Mol Genet 19:R131R136 (2010).

Mundlos S: The brachydactylies: a molecular disease family. Clin Genet 76:123-136 (2009).

-Polinkovsky A, Robin NH, Thomas JT, Irons M, Lynn A, et al: Mutations in CDMP1 cause autosomal dominant brachydactyly type C. Nat Genet 17:18-19 (1997).

Roy A, Kucukural A, Zhang Y: I-TASSER: a unified platform for automated protein structure and function prediction. Nat Protoc 5:725738 (2010).
- Seemann P, Schwappacher R, Kjaer KW, Krakow $\mathrm{D}$, Lehmann K, et al: Activating and deactivating mutations in the receptor interaction site of GDF5 cause symphalangism or brachydactyly type A2. J Clin Invest 115:2373-2381 (2005).

eemann P, Brehm A, König J, Reissner C, Stricker S, et al: Mutations in GDF5 reveal a key residue mediating BMP Inhibition by NOGGIN. PloS Genet 5:e1000747 (2009).

Szczaluba K, Hilbert K, Obersztyn E, Zabel B, Mazurczak T, Kozlowski K: Du Pan syndrome phenotype caused by heterozygous pathogenic mutations in CDMP1 gene. Am J Med Genet A 138:379-383 (2005).

Tang B, Nirasawa S, Kitaoka M, Marie-Claire C, Hayashi K: General function of N-terminal propeptide on assisting protein folding and inhibiting catalytic activity based on observations with a chimeric thermolysin-like protease. Biochem Bioph Res Co 301:1093-1098 (2003).
Temtamy SA, Aglan MS: Brachydactyly. Orphanet J Rare Dis 3:15 (2008).

Tsumaki N, Tanaka K, Arikawa-Hirasawa E, Nakase T, Kimura T, et al: Role of CDMP-1 in skeletal morphogegesis: promotion of mesenchymal cell recruitment and chondrocyte differentiation. J Cell Biol 144:161-173 (1999).

Yang W, Cao L, Liu W, Jiang L, Sun M, et al: Novel point mutations in GDF5 associated with two distinct limb malformations in Chinese: brachydactyly type $\mathrm{C}$ and proximal symphalangism. J Hum Genet 53:368-374 (2008).

Zhang Y: I-TASSER server for protein 3D structure prediction. BMC Bioinformatics 9:40 (2008). 